\title{
Searching for doubly charged Higgs bosons in Möller scattering by resonance effects at linear $e^{-} e^{-}$collider
}

\author{
Chian-Shu Chen*, Chao-Qiang Geng ${ }^{\dagger}$, and Dmitry V. Zhuridov ${ }^{\ddagger}$ \\ Department of Physics, National Tsing Hua University, Hsinchu, Taiwan 300
}

(Dated: January 1, 2019)

\begin{abstract}
We discuss the parity-violating left-right asymmetries (LRAs) in Möller scattering at the International Linear Collider (ILC) induced by doubly charged Higgs bosons in models with $S U(2)_{L}$ triplet and singlet scalar bosons, which couple to the left- and right-handed charged leptons, respectively. These bosons are important in scenarios for the generation of the neutrino mass. We demonstrate that the contributions to the LRAs from the triplet and singlet bosons are opposite to each other. In particular, we show that the doubly charged Higgs boson from the singlet scalar can be tested at the ILC by using the resonance effect.
\end{abstract}

\footnotetext{
* e-mail: chianshu@gmail.com

$\dagger$ e-mail: geng@phys.nthu.edu.tw

‡e-mail: zhuridov@phys.nthu.edu.tw
} 
The smallness of active neutrino masses is one of the most challenging problems in particle physics. The many interesting scenarios proposed to explain it can be divided into the categories of ones with and without the right-handed neutrinos (RNs). For examples, the small neutrino masses can be generated through the widely known seesaw mechanism $[1,2]$ with the RNs embedded in new physics at a high energy scale, such as grand unified theories [3] and extra dimensions [4], while models with extended Higgs sectors can accomplish the goal without the RNs $[5,6,7,8,9,10,11]$. The former is difficult to test directly due to the fact that the RNs have no standard-model (SM) interactions and the latter gets many interesting features, such as the existence of the doubly charged Higgs bosons $H^{ \pm \pm}$from the triplet (singlet) coupling to the left- (right-) handed charged leptons to generate the neutrino masses at tree [7] (two-loop) level [6, 9, 10, 11] radiatively.

In this paper, we show that it is possible to distinguish the models with these two types of $H^{ \pm \pm}$by studying Möller scattering [12] of $e^{-} e^{-} \rightarrow e^{-} e^{-}$at a linear $e^{-} e^{-}$collider, such as the International Linear Collider (ILC) [13, 14]. With polarized initial electron beams, one can define the parity-violating left-right asymmetry (LRA) [15] by

$$
A_{P}=\frac{\frac{d \sigma_{L L}}{d \cos \theta}-\frac{d \sigma_{R R}}{d \cos \theta}}{\frac{d \sigma_{L L}}{d \cos \theta}+\frac{d \sigma_{R R}}{d \cos \theta}},
$$

where $L L$ and $R R$ denote the initial $e^{-} e^{-}$polarizations, and $\theta$ is the angle between the initial and final ee beams. As the asymmetry can be measured with high accuracy in the process, it provides an excellent opportunity to probe new-physics effects. In particular, we will demonstrate that the LRA is sensitive to the doubly charged Higgs bosons of $H^{ \pm \pm}$at their mass poles.

We start by considering a complex triplet scalar $H_{L} \equiv T_{(2)}$ with the subscript denoting the hypercharge, which couples to the $S U(2)_{L}$ lepton doublets $\left(L_{i L}\right)$ [7]:

$$
\mathcal{L}_{L}=g_{i j} \overline{L_{i L}^{c}} T L_{j L}+\text { H.c. },
$$

and a singlet scalar $H_{R} \equiv \Psi_{(4)}$ which couples to the $S U(2)_{L}$ charged lepton singlets $\left(\ell_{i R}\right)[9$, 10, 11]:

$$
\mathcal{L}_{R}=Y_{i j} \overline{\ell_{i R}^{c}} \ell_{j R} \Psi+\text { H.c. },
$$

where $g_{i j}$ and $Y_{i j}$ are the coupling constants, $i, j=e, \mu, \tau$, and $c$ stands for charge conjugation. The contributions in the SM and those with $H_{\alpha}^{--}(\alpha=L, R)$ to the Möller scattering at tree level are shown in Fig. 1. 

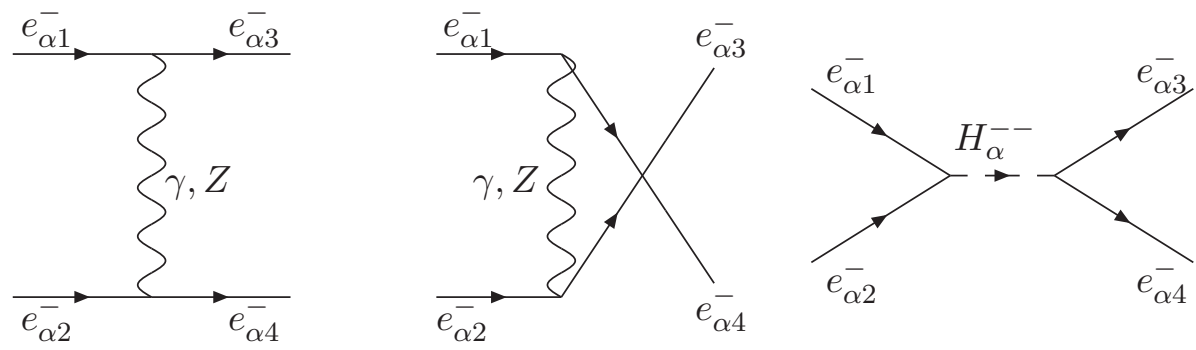

FIG. 1: Feynman diagrams of the Möller scattering for the contributions from $\gamma, Z$ and $H_{\alpha}^{--}$.

In the SM [16], the matrix elements for Möller scattering due to $\gamma$ and $Z$ are given by

$$
\begin{aligned}
M_{\gamma \alpha} & =\frac{e^{2}}{t}\left(\bar{e}_{3} \gamma^{\mu} P_{\alpha} e_{1}\right)\left(\bar{e}_{4} \gamma^{\mu} P_{\alpha} e_{2}\right)-\frac{e^{2}}{u}\left(\bar{e}_{4} \gamma^{\mu} P_{\alpha} e_{1}\right)\left(\bar{e}_{3} \gamma^{\mu} P_{\alpha} e_{2}\right), \\
M_{Z \alpha} & =\frac{C_{\alpha}^{2}}{\tilde{t}}\left(\bar{e}_{3} \gamma^{\mu} P_{\alpha} e_{1}\right)\left(\bar{e}_{4} \gamma^{\mu} P_{\alpha} e_{2}\right)-\frac{C_{\alpha}^{2}}{\tilde{u}}\left(\bar{e}_{4} \gamma^{\mu} P_{\alpha} e_{1}\right)\left(\bar{e}_{3} \gamma^{\mu} P_{\alpha} e_{2}\right),
\end{aligned}
$$

where $P_{L(R)}=\frac{1}{2}\left(1 \mp \gamma_{5}\right), a=t, u$ is for the Mandelstam variables, $\tilde{a}=a-M_{Z}^{2}$ and

$$
C_{L}=\frac{g\left(1-2 \sin ^{2} \theta_{W}\right)}{2 \cos \theta_{W}}, \quad C_{R}=\frac{g \sin ^{2} \theta_{W}}{\cos \theta_{W}} .
$$

The non-SM interactions in Eqs. (2) and (3) can be combined into a single form with the doubly charged Higgs bosons $H_{\alpha}^{++}$and $i=j=e$ as follows:

$$
\mathcal{L}_{H_{\alpha}}=Y_{\alpha e e} \overline{e^{c}} P_{\alpha} e H_{\alpha}^{++}+\text {H.c. },
$$

which leads to

$$
M_{H_{\alpha}}=\left|Y_{\alpha e e}\right|^{2}\left(\bar{e}_{2}^{c} P_{\alpha} e_{1}\right) \frac{1}{s-M_{H_{\alpha}}^{2}-i M_{H_{\alpha}} \Gamma_{H_{\alpha}}}\left(\bar{e}_{4} P_{\alpha} e_{3}^{c}\right),
$$

with the total widths $[10]$

$$
\begin{aligned}
& \Gamma_{T}=3\left[\Gamma_{L}\left(\ell_{i}^{ \pm} \ell_{i}^{ \pm}\right)+\Gamma_{L}\left(\ell_{i}^{ \pm} \ell_{j}^{ \pm}\right)_{i \neq j}\right]+\Gamma_{L}\left(W^{ \pm} W^{ \pm}\right)+\Gamma_{L}\left(W^{ \pm} P^{ \pm}\right)+\Gamma_{L}\left(W^{ \pm} W^{ \pm} T_{a}^{0}\right), \\
& \Gamma_{\Psi}=3\left[\Gamma_{R}\left(\ell_{i}^{ \pm} \ell_{i}^{ \pm}\right)+\Gamma_{R}\left(\ell_{i}^{ \pm} \ell_{j}^{ \pm}\right)_{i \neq j}\right],
\end{aligned}
$$

where $Y_{\alpha e e}$ are new coupling constants related to $g_{e e}$ and $Y_{e e}$, and

$$
\begin{aligned}
\Gamma_{\alpha}\left(\ell_{i}^{ \pm} \ell_{j}^{ \pm}\right) & =\left(2-\delta_{i j}\right) \frac{\left|Y_{\alpha i j}\right|^{2}}{16 \pi} M_{H_{\alpha}} \\
\Gamma_{L}\left(W^{ \pm} W^{ \pm}\right) & =\frac{g^{4} v_{T}^{2}}{16 \pi M_{T}} \sqrt{1-\frac{4 M_{W}^{2}}{M_{T}^{2}}}\left(3-\frac{M_{T}^{2}}{M_{W}^{2}}+\frac{M_{T}^{2}}{4 M_{W}^{2}}\right), \\
\Gamma_{L}\left(W^{ \pm} P^{ \pm}\right) & =\frac{g^{2} M_{T}^{3}}{16 \pi M_{W}^{2}} \lambda^{3 / 2}\left(1, \frac{M_{W}^{2}}{M_{T}^{2}}, \frac{M_{P}^{2}}{M_{T}^{2}}\right),
\end{aligned}
$$


with $\lambda(x, y, z)=x^{2}+y^{2}+z^{2}-2 x y-2 x z-2 y z$ and $P^{ \pm}$and $T_{a}^{0}$ being the single-charged and neutral components of the Higgs scalars, respectively. Note that the subscript $a$ in $T_{a}^{0}$ denotes the mass eigenstate, which is a mixture of the doublet and triplet bosons. The three-body decay modes in Eq. (8) are expected to be relatively suppressed by the phase space compared to the two-body ones. Note that we have assumed the particular model to include either one of the two new interactions or both. Since the triplet scalar contains the SM quantum numbers, the gauge interactions provide more decay channels for its doubly charged component.

In the scenario with the interaction in Eq. (2), the neutrino masses are proportional to $g_{i j} v_{T}$, where $v_{T}$ denotes the VEV of $T$. However, from the neutrino oscillation data and cosmological experiments there are restricting bounds on the neutrino masses, given by [17]

$$
m_{\nu} \sim g_{i j} v_{T} \lesssim 0.1 \mathrm{eV}
$$

On the other hand, the precision data of $\rho=1.0002_{-0.0004}^{+0.0007}$ [16] result in a limit of $v_{T}$ that is less than $4.41 \mathrm{GeV}$ [10]. We shall use $v_{T}=4 \mathrm{GeV}$ in our numerical calculations.

The parity-violating LRA in Eq. (1) can now be rewritten as

$$
A_{P}=\frac{\sum_{\text {spin }}\left|M_{L}\right|^{2}-\sum_{\text {spin }}\left|M_{R}\right|^{2}}{\sum_{\text {spin }}\left|M_{L}\right|^{2}+\sum_{\text {spin }}\left|M_{R}\right|^{2}},
$$

where

$$
\sum_{\text {spin }}\left|M_{\alpha}\right|^{2}=\sum_{\text {spin }}\left|M_{\alpha}\right|_{S M}^{2}+\sum_{\text {spin }}\left|M_{H \alpha}\right|^{2}
$$

In Eq. (12), the SM contributions are

$$
\sum_{\text {spin }}\left|M_{\alpha}\right|_{S M}^{2}=\sum_{\text {spin }}\left|M_{\gamma \alpha}\right|^{2}+\sum_{\text {spin }}\left|M_{Z \alpha}\right|^{2}+\left(\sum_{\text {spin }} M_{\gamma \alpha} M_{Z \alpha}^{\dagger}+\sum_{\text {spin }} M_{Z \alpha} M_{\gamma \alpha}^{\dagger}\right)
$$

where

$$
\begin{gathered}
\sum_{\text {spin }}\left|M_{\gamma \alpha}\right|^{2}=\left[2 e^{2} \frac{s(t+u)}{t u}\right]^{2}, \quad \sum_{\text {spin }}\left|M_{Z \alpha}\right|^{2}=\left[2 C_{\alpha} \frac{s(\tilde{t}+\tilde{u})}{\tilde{t} \tilde{u}}\right]^{2} \\
\sum_{\text {spin }} M_{\gamma \alpha} M_{Z \alpha}^{\dagger}=\sum_{\text {spin }} M_{Z \alpha} M_{\gamma \alpha}^{\dagger}=4 e^{2} C_{\alpha} s^{2}\left[\frac{1}{t \tilde{t}}+\frac{1}{t \tilde{u}}+\frac{1}{u \tilde{t}}+\frac{1}{u \tilde{u}}\right]
\end{gathered}
$$


and the non-SM ones due to the doubly charged Higgs bosons are

$$
\sum_{\text {spin }}\left|M_{H \alpha}\right|^{2}=\left|Y_{\alpha e e}\right|^{4} \frac{s^{2}}{\left(s-M_{H_{\alpha}}^{2}\right)^{2}+M_{H_{\alpha}}^{2} \Gamma_{H_{\alpha}}^{2}} .
$$

An interesting point here is that the interference between the SM and the new interactions, i.e., the vector-scalar interference term, vanishes in this calculation.
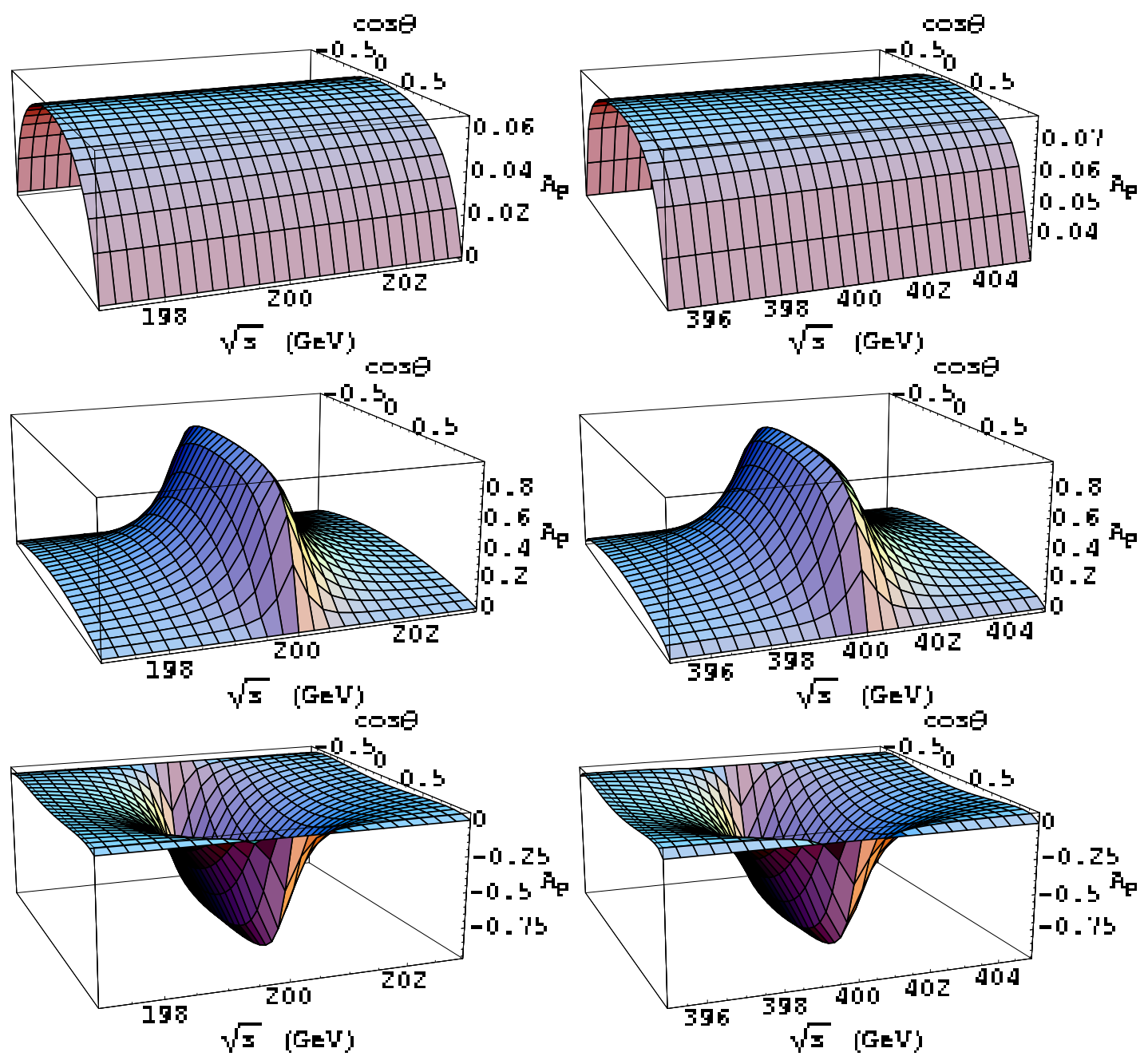

FIG. 2: $A_{P}$ versus $\cos \theta$ for the energies around $\sqrt{s}=200 \mathrm{GeV}$ (left) and $400 \mathrm{GeV}$ (right) in the SM (upper) and the models with the triplet (middle) and singlet (lower) scalar bosons, where $M_{H_{\alpha}}=200 \mathrm{GeV}$ (left) and $400 \mathrm{GeV}$ (right), respectively.

The results for $A_{P}$ versus $\cos \theta$ are presented in Fig. 2 for the SM and the models with the triplet and singlet bosons in the interactions of Eqs. (2) and (3), respectively. In the figures (middle and lower), the resonance energies for the non-SM cases are considered, the doubly charged Higgs boson masses are taken to be $M_{H \alpha}=200$ (left) and $400 \mathrm{GeV}$ (right) 
with the couplings $\left|Y_{\alpha e e}\right|=0.1$ close to the upper bounds [10], and the values of $\Gamma_{H \alpha}$ are given in Table I. The current lower bound on the doubly charged scalar mass set by the

TABLE I: The values of $\Gamma_{H \alpha}$ for the various values of $M_{H \alpha}$.

\begin{tabular}{|c|c|c|c|}
\hline$\left|Y_{\alpha e e}\right|$ & $M_{H \alpha}, \mathrm{GeV}$ & $\Gamma_{T}, \mathrm{GeV}$ & $\Gamma_{\Psi}, \mathrm{GeV}$ \\
\hline 0.1 & 200 & 0.359 & 0.358 \\
\hline 0.1 & 400 & 0.736 & 0.716 \\
\hline 0.05 & 200 & 0.091 & 0.090 \\
\hline 0.02 & 200 & 0.015 & 0.014 \\
\hline 0.005 & 200 & 0.0020 & 0.00090 \\
\hline 0.002 & 200 & 0.0012 & 0.00014 \\
\hline
\end{tabular}

direct search at the Tevatron in Fermilab is $136 \mathrm{GeV}$ [18]. Since the doubly charged Higgs bosons in Eqs. (2) and (3) couple to the different helicity states of the electron, the effects lead to an opposite sign of $A_{P}$. Thus, one can distinguish the two types of the interactions based on the sign of $A_{P}$ around the resonance.

It should be stressed that these effects are strongly dependent on the values of the couplings $Y_{\alpha e e}$, since the non-SM effects are proportional to $\left|Y_{\alpha e e}\right|^{4}$, and the widths $\Gamma_{\alpha}\left(e^{ \pm} e^{ \pm}\right)$ are proportional to $\left|Y_{\alpha e e}\right|^{2}$. This is shown for the resonance point of $\sqrt{s}=M_{H \alpha}=200 \mathrm{GeV}$ and the near one of $\sqrt{s}=202 \mathrm{GeV}$ in Fig. 3. By taking into account the strong bounds in Eq. (10) on the couplings $g_{i j} \sim Y_{L i j}$ in Eq. (15), one may conclude that there is no chance to observe the LRA due to the interaction in Eq. (2) for the triplet scalar in the near future. On the other hand, the constraints on the interaction in Eq. (3) for the singlet scalar are much relaxed [10], providing a good opportunity to detect the effects at the ILC in its $e^{-} e^{-}$ mode [19]. As illustrated in Fig. 3, in the case of $\Psi$ there is not precisely a dependence on $\left|Y_{\alpha e e}\right|$ in the resonance point of $\sqrt{s}=M_{H \alpha}$. This is because the factor $\left|Y_{\alpha e e}\right|^{4}$ in the numerator and the factor $\left|Y_{\alpha e e}\right|^{2}$ in the width cancel each other.

We now consider the models that include the complex triplet and singlet bosons, simultaneously. In this kind of models, the two doubly charged Higgs bosons mix to each other with the mixing angle $\delta$ to form the mass eigenstates $P_{1,2}$ :

$$
\left(\begin{array}{c}
P_{1}^{ \pm \pm} \\
P_{2}^{ \pm \pm}
\end{array}\right)=\left(\begin{array}{cc}
\cos \delta & \sin \delta \\
-\sin \delta & \cos \delta
\end{array}\right)\left(\begin{array}{c}
T^{ \pm \pm} \\
\Psi^{ \pm \pm}
\end{array}\right)
$$



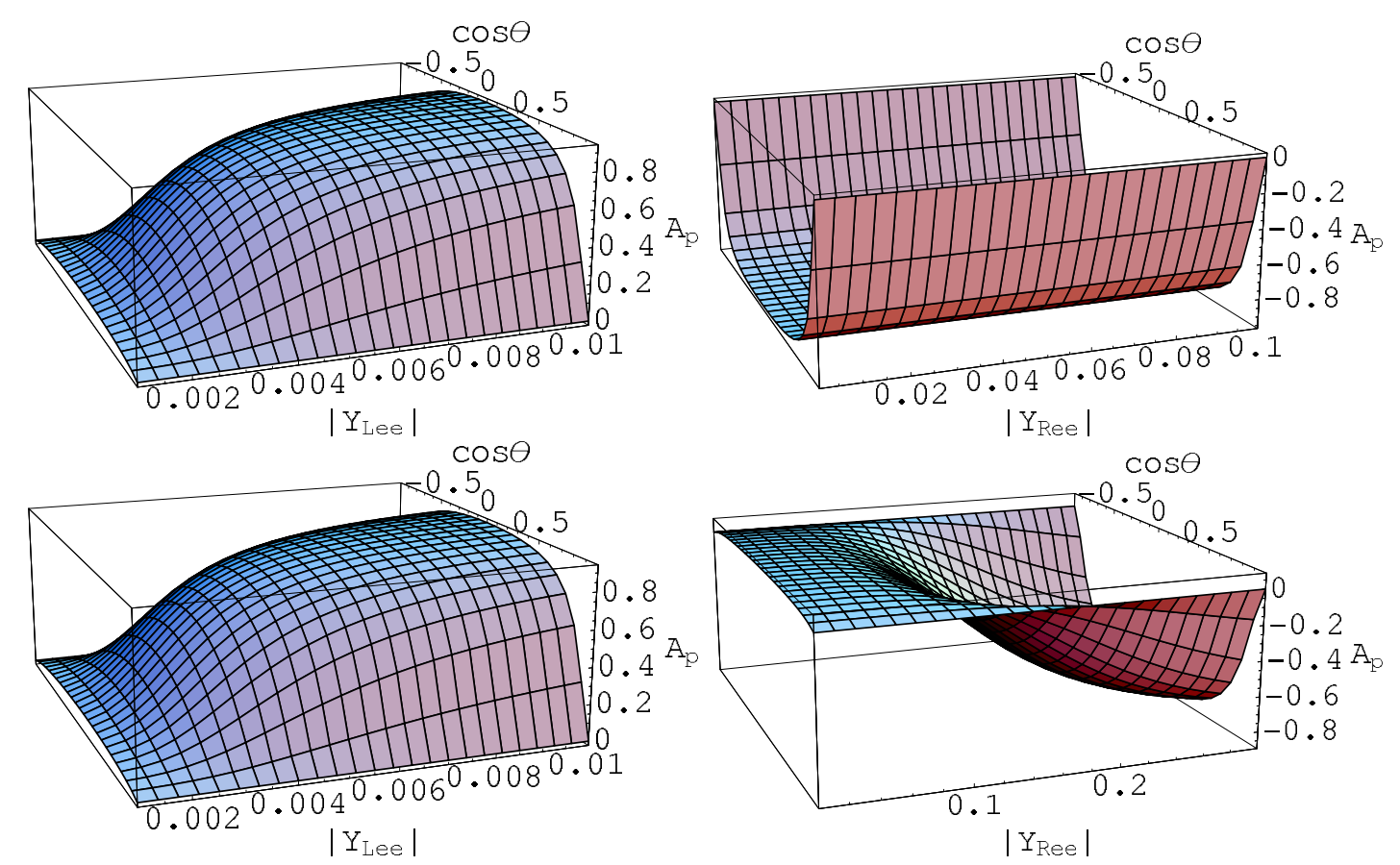

FIG. 3: $A_{p}$ versus $\cos \theta$ and $\left|Y_{\alpha e e}\right|$ in the resonance point $\sqrt{s}=M_{H \alpha}=200 \mathrm{GeV}$ (upper), the near one of $\sqrt{s}=202 \mathrm{GeV}$ (lower) with the doubly charged Higgs bosons from the triplet (left) and singlet (right) scalars.

Therefore, the interactions in Eqs. (2) and (3) can be rewritten as

$$
\begin{aligned}
\mathcal{L}_{L M} & =g_{i j} \overline{\ell_{L i}^{c}} \ell_{L j}\left(\cos \delta P_{1}-\sin \delta P_{2}\right)+\text { H.c. }, \\
\mathcal{L}_{R M} & =Y_{i j} \overline{\ell_{R i}^{c}} \ell_{R j}\left(\sin \delta P_{1}+\cos \delta P_{2}\right)+\text { H.c. },
\end{aligned}
$$

which become the pure left- and right-handed interactions for the limit $\delta \rightarrow 0$, respectively. Here, we will concentrate on the lighter massive state $P_{1}$. The considered models share both features of the models with only the interaction in Eq. (2) or (3). Due to the strong constraint on the couplings in Eq. (2), these models can give a significant contribution only to $d \sigma_{R R}$ in Eq. (1), which tends to flip the sign of $A_{P}$. On the other hand all the decay channels in Eq. (8) are permitted for $P_{1}$ due to the mixing in the doubly charged Higgs sector.

In the model proposed in Refs. $[9,10]$, as there is no tree-level triplet Yukawa interaction in Eq. (2), only the singlet interaction in Eq. (3) is allowed. In terms of the triplet gauge couplings and singlet Yukawa couplings the decay channels $P_{1}^{ \pm \pm} \rightarrow \ell_{i R}^{ \pm} \ell_{j R}^{ \pm}, P_{1}^{ \pm \pm} \rightarrow W^{ \pm} W^{ \pm}$, $P_{1}^{ \pm \pm} \rightarrow W^{ \pm} P^{ \pm}$and $P_{1}^{ \pm \pm} \rightarrow W^{ \pm} W^{ \pm} T_{a}^{0}$ are permitted. We remark that the widths of these 
decays have an additional mixing-angle dependence compared with those in Eq. (9) [10]. As shown in Fig. 4, the deviations from the SM, allowed by the present experimental bounds, can be observed at the ILC. In our discussion, we have taken the upper bound of $\left|Y_{e e}\right|=0.2$ [10] and the small (large) mixing of $\sin \delta=0.1(0.5)$. The values of $\Gamma_{P_{1}}$ are given in Table II.

TABLE II: The values of $\Gamma_{P_{1}}$ for $M_{P_{1}}=200$ and $400 \mathrm{GeV}$ and $\sin \delta=0.1$ and 0.5 .

\begin{tabular}{|c|c||c|}
\hline $\sin \delta$ & $M_{P_{1}}, \mathrm{GeV}$ & $\Gamma_{P_{1}}, \mathrm{GeV}$ \\
\hline \hline 0.1 & 200 & 0.015 \\
& 400 & 0.048 \\
\hline 0.5 & 200 & 0.359 \\
& 400 & 0.732 \\
\hline
\end{tabular}
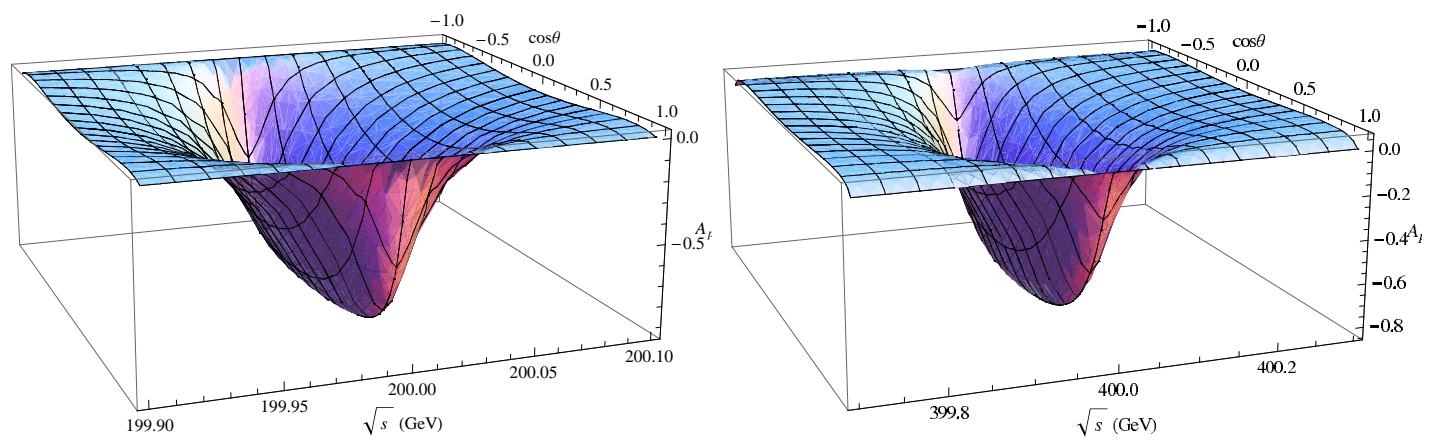

FIG. 4: $A_{P}$ in the general model at the resonance points of $200 \mathrm{GeV}$ (left) and $400 \mathrm{GeV}$ (right) with small mixing and large mixing (see separate files alongside the text) angles.

The valleys in Fig. 4 are much wider in the case with the large mixing than those with the small mixing. This can help us to clarify the mixing between the doubly charged scalars. For example, Fig. 5 shows that it is easy to separate the model with the large mixing by using the $A_{P}$ versus $\sqrt{s}$ dependence, while models with the small mixing and pure singlet (no mixing) are practically undistinguishable. Measuring of the width and height of the resonance could determine $Y_{\alpha e e}$ and $\sin \delta$, which are relevant to the neutrino mixings.

The relative deviations of $A_{P}$ from the SM values of $A_{P}^{S M}$, defined by $\alpha_{P}=\left(A_{P}^{S M}-\right.$ $\left.A_{P}\right) / A_{P}^{S M}$ are given in Table III for various resonance energies $\sqrt{s}$ at the maximum points of $\cos \theta=0$ with $M_{P_{1}}=200$ and $400 \mathrm{GeV}$ and $\sin \delta=0.1$ and 0.5 . One can see that measuring $\alpha_{P}$ with $10 \%$ (1\%) accuracy and fixing the energy with $1 \%$ accuracy or less allows one to 


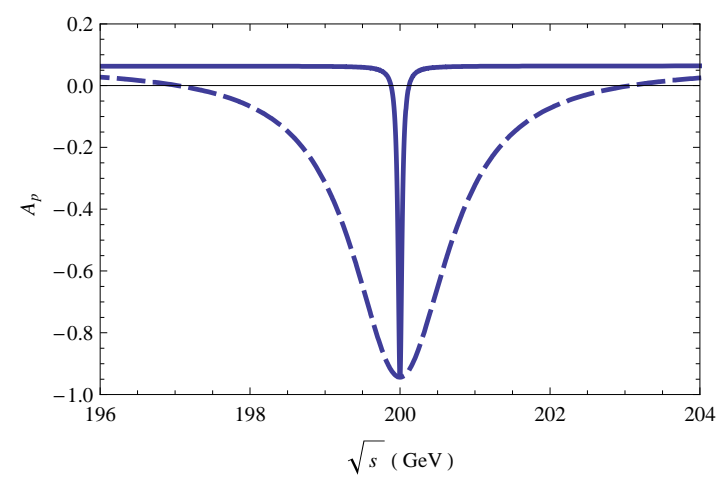

FIG. 5: $A_{P}$ versus $\sqrt{s}$ for $M_{P_{1}}=200 \mathrm{GeV}$ for pure singlet and small mixing (coincided solid lines), large mixing (dashed line).

observe the resonance effects in the large (small) mixing. This accuracy can be achieved with realistic polarizations for the initial electrons, since the beam energy and polarizations are expected to be stable and measurable at a level of about $0.1 \%$ [13]. Taking the electron longitudinal polarizations of 0.8 and assuming their systematic uncertainties of $0.5 \%$ [19], we estimate that the corresponding uncertainty in measuring $A_{P}$ is about $1 \%$. At the resonance, the non-standard contributions to the LRA are dominant. The dependence on the mixing angle in Eq. (11) almost disappears since the terms with $Y_{L i j}$ are small. For this reason, $\alpha_{P}$ is about the same for the different mixings at the resonance.

The integrated deviations $\tilde{\alpha}_{P}=\left(\tilde{A}_{P}^{S M}-\tilde{A}_{P}\right) / \tilde{A}_{P}^{S M}$, with

$$
\tilde{A}_{P}=\frac{\sigma_{L L}-\sigma_{R R}}{\sigma_{L L}+\sigma_{R R}}
$$

are given in the last three columns in Table III for the two types of mixing and the pure singlet $(\sin \theta=1)$. In Eq. (18), we have integrated the differential cross sections over $\cos \theta$ in the range $\cos 1^{\circ}$ to $\cos 179^{\circ}$, since coverage down to $\sim 1^{\circ}$ from the beam axis is expected at ILC [14]. From Table III, we see that $\tilde{\alpha}_{P}$ decreases with increasing $M_{P_{1}}$ and slightly depends on the mixing angle.

In conclusion, we have investigated the contributions to the LRAs from the doubly charged Higgs bosons in models with triplet and singlet scalar bosons, which couple to the electrons with different chiralities in Möller scattering, respectively. We have found that it is easy to extract the properties of the models with $H^{ \pm \pm}$interacting with $e_{R}$ from the measurements of $A_{P}$ around the resonance point at the ILC, whereas it is hard to observe the effects of the models with $H^{ \pm \pm}$coupled only to $e_{L}$. As the models have different mechanisms 
TABLE III: Relative deviations $\alpha_{P}$ of $A_{P}$ at $\cos \theta=0$ for the various $M_{P_{1}}$ and mixings $s_{\delta} \equiv \sin \delta$ in and around the resonance energies. The integrated deviations $\tilde{\alpha}_{P}$ of $\tilde{A}_{P}$ are also given.

\begin{tabular}{|c|c||c|c||c|c|c|}
\hline$M_{P_{1}}, \mathrm{GeV}$ & $\sqrt{s}, \mathrm{GeV}$ & $\alpha_{P}, s_{\delta}=0.1$ & $\alpha_{P}, s_{\delta}=0.5$ & $\tilde{\alpha}_{P}, s_{\delta}=0.1$ & $\tilde{\alpha}_{P}, s_{\delta}=0.5$ & $\tilde{\alpha}_{P}, s_{\delta}=1$ \\
\hline \hline 200 & 200 & 0.47 & 0.47 & 38.0 & 41.4 & 41.6 \\
& $200 \pm 1$ & 0.015 & 0.46 & & & \\
& $200 \pm 2$ & 0.004 & 0.41 & & & \\
& $200 \pm 4$ & 0.001 & 0.28 & & & \\
\hline \multirow{2}{*}{400} & 400 & 0.33 & 0.35 & 5.5 & 13.5 & 14.1 \\
& $400 \pm 1$ & 0.039 & 0.35 & & & \\
& $400 \pm 2$ & 0.012 & 0.34 & & & \\
& $400 \pm 4$ & 0.003 & 0.30 & & & \\
\hline
\end{tabular}

for the generation of the neutrino mass, future searches for $A_{P}$ at the ILC are important for us to understand the neutrino physics. Finally, we remark that the $H^{ \pm \pm}$masses for the resonance studies at the ILC can be determined by the searches at the Large Hadron Collider (LHC), such as the dilepton signatures at the LHC shown in Ref. [11].

\section{Acknowledgements}

This work is financially supported by the National Science Council of Republic of China under the contract \#: NSC-95-2112-M-007-059-MY3. One of us (DVZ) would like to thank TILK08 for hospitality in Sendai where this research was presented.

[1] P. Minkowski, Phys. Lett. B 67 (1977) 421; T. Yanagida, in Proceedings of the Workshop on the Unified Theory and the Baryon Number in the Universe, eds. O. Sawada and A. Sugamoto (Tsukuba, Japan, 1979) 95; M. Gell-Mann, P. Ramond, and R. Slansky, in Supergravity, eds. D.Z. Freedman and P.van Nieuwenhuizen (North Holland, Amsterdam, 1979) 315; S. L. Glashow, in Proceedings of the 1979 Cargese Summer Instute on Quarks and Leptons, eds. M. Levy et al. (New York, 1980) 687; R.N. Mohapatra and G. Senjanovic, Phys. Rev. 
Lett. 44 (1980) 912.

[2] B. Kayser, F. Gibrat-Debu, and F. Perrier, The Physics of the Massive Neutrinos, World Scientific, Singapore (1989); R.N. Mohapatra et al., Rept. Prog. Phys. 70 (2007) 1757.

[3] R. Hempfling, Nucl. Phys. B 478 (1996) 3; Y. Nomura and T. Yanagida, Phys. Rev. D 59 (1999) 017303.

[4] K. R. Dienes, E. Dudas and T. Gherghetta, Nucl. Phys. B 557 (1999) 25; Y. Grossman and M. Neubert, Phys. Lett. B 474 (2000) 361; S. J. Huber and Q. Shafi, Phys. Lett. B 583 (2004) 293.

[5] A. Zee, Phys. Lett. B 93 (1980) 389 [Erratum ibid. B 95 (1980) 461]; Phys. Lett. B 161 (1985) 141.

[6] A. Zee, Nucl. Phys. B 264 (1986) 99; K. S. Babu, Phys. Lett. B 203 (1988) 132.

[7] W. Konetschny and W. Kummer, Phys. Lett. B 70 (1977) 433; J. Schechter and J. W. F. Valle, Phys. Rev. D 22 (1980) 2227; T. P. Cheng and L. F. Li, Phys. Rev. D 22 (1980) 2860; G. Lazarides, Q. Shafi, C. Wetterich, Nucl. Phys. B 181 (1981) 287; R.N. Mohapatra and G. Senjanovic, Phys. Rev. D 23 (1981) 165.

[8] J. Garayoa and T. Schwetz, arXiv: 0712.1453 [hep-ph].

[9] C. S. Chen, C. Q. Geng and J. N. Ng, Phys. Rev. D 75 (2007) 053004.

[10] C. S. Chen, C. Q. Geng, J. N. Ng and J. M. S. Wu, JHEP 0708 (2007) 022.

[11] C. S. Chen, C. Q. Geng and D. V. Zhuridov, Phys. Lett. B 666 (2008) 340.

[12] C. Möller, Ann. der Physik 14 (1932) 531; M. J. Puhala, T. G. Rizzo and B. L. Young, Phys. Lett. B 109 (1982) 411; T. G. Rizzo, Phys. Rev. D 25 (1982) 1355; B. Mukhopadhyaya, S. K. Rai, Phys. Lett. B 633 (2006) 519.

[13] J. Brau et al. (ILC Collab), arXiv: 0712.1950 [physics.acc-ph].

[14] LHC/LC Study Group: G. Weiglein et al., Phys. Rept. 426 (2006) 47; J. A. Aguilar-Saavedra et al. [ECFA/DESY LC Physics Working Group], arXiv:hep-ph/0106315; K. Abe et al. [ACFA Linear Collider Working Group], arXiv:hep-ph/0109166.

[15] A. Czarnecki and W. J. Marciano, Int. J. Mod. Phys. A 15 (2000) 2365; F. Cuypers and P. Gambino, Phys. Lett. B 388 (1996) 211; P. L. Anthony et al., Phys. Rev. Lett. 92 (2004) 181602.

[16] W. M. Yao et al. (Particle Data Group), J. Phys. G 33 (2006) 1.

[17] P. F. Perez, T. Han, T. Li, M. J. Ramsey-Musolf, arXiv: 0810.4138 [hep-ph]; I. Tereno et al., 
arXiv: 0810.0555 [astro-ph]; M. C. Gonzalez-Garcia, M. Maltoni, Phys. Rept. 460 (2008) 1. [18] D. Acosta et al. (CDF Collab.), Phys. Rev. Lett. 93 (2004) 221802.

[19] C. A. Heusch, Int. J. Mod. Phys. A 20 (2005) 7289; A. A. Pankov and N. Paver, Int. J. Mod. Phys. A 20 (2005) 7392. 